\title{
Unusual Bound or Localized States
}

\author{
M. A. Cirone ${ }^{\mathrm{a}}$, G. Metikas ${ }^{\mathrm{a}}$, and W. P. Schleich ${ }^{\mathrm{a}, \mathrm{b}}$ \\ ${ }^{a}$ Abteilung für Quantenphysik, Universität Ulm, D-89069 Ulm, Germany \\ ${ }^{b}$ Department of Physics, North Texas State University, Denton, TX \\ Reprint requests to Dr. M. A. C. E-mail: m_cirone@virgilio.it
}

Z. Naturforsch. 56 a, 48-60 (2001); received February 11, 2001

Presented at the 3rd Workshop on Mysteries, Puzzles and Paradoxes in Quantum Mechanics, Gargnano, Italy, September 17 - 23, 2000.

\begin{abstract}
We summarize unusual bound or localized states in quantum mechanics. Our guide through these intriguing phenomena is the classical physics of the upside-down pendulum, taking advantage of the analogy between the corresponding Newton's equation of motion and the time independent Schrödinger equation. We discuss the zero-energy ground state in a three-dimensional, spatially oscillating, potential. Moreover, we focus on the effect of the attractive quantum anti-centrifugal potential that only occurs in a two-dimensional situation.
\end{abstract}

Key words: Quantum Mechanics; Bound States; Parametric Oscillator; Periodic Potential.

\section{The Pendulum: A Guide to Quantum Physics}

Determining the energy levels of a quantum system was a desperate enterprise before the advent of the Bohr-Sommerfeld quantization conditions. Max Planck had emphasized that the energy of the harmonic oscillator is quantized in units of a fundamental energy given by the product of what we now call Dirac's constant and the frequency of the oscillator. However, this rule failed miserably when used in other quantum systems. Paul Ehrenfest's adiabatic principle, applied to the pendulum [1] whose length slowly changes as a function of time, made clear that it is not the energy but the action that is quantized, but why? When we change the length of the pendulum adiabatically, the amplitude of oscillation does not stay constant, neither does the frequency nor the energy. What stays constant is the action, that is, the area in phase space. For the founding fathers of quantum mechanics it must have been a miracle, an amazing fact symbolizing in post-Schrödinger language that the number of nodes in an energy wave function stays constant under adiabatic changes.

The classical dynamics of the pendulum serves as an excellent guide for many quantum phenomena. For example, the upside-down pendulum yields insight into the energy wave function of a periodic potential [2]. Here we do not focus on the familiar Bloch states which appear when the potential enjoys a strict periodicity over the whole space. Our states occur when the modulation of the potential extends only over a finite domain of space. Due to the shape of this potential we refer to it as the accordion potential. Extensions of these one-dimensional considerations to two and three dimensions lead us to the effect of the quantum anti-centrifugal force [3].

In the present paper we take seriously the joke 'physics takes mathematics and makes it understandable', therefore we shall suppress all the mathematics and highlight the essential ideas. This approach is justified by the fact that we are not inventing or applying new mathematics, but attempt to draw together phenomena of different fields exposing a common thread. We shall focus on a general point of view but emphasize that the modern tools of cold atoms in a standing electromagnetic wave can demonstrate these unusual bound or localized states predicted in this paper.

We follow one 'Leitmotiv', that is, a theme common to all phenomena discussed in this article: The classical physics of the upside-down pendulum. Moreover, we develop a little side theme summarized by the phrase "wave mechanics in quantum potentials".

Our paper is organized as follows: In Sect. 2 we briefly summarize the classical physics of the upsidedown pendulum and then turn in Sect. 3 to related 
phenomena, such as the Paul trap, the helium atom and field induced multipoles. We devote Sect. 4 to the transition to quantum mechanics, and in particular discuss the shape of the ground state wave function in the accordion potential. In Sect. 5 we show that in three dimensions a localized ground state can exist - even at zero energy. The quantum anti-centrifugal potential emerges when in Sect. 6 we focus on the Schrödinger equation in two dimensions. We conclude in Sect. 7 with an outlook and present extensions of the concepts discussed in this paper. An Appendix summarizes the mathematical aspects of the upside-down pendulum and the Schrödinger equation of the particle in a periodic potential.

\section{The Upside-down Pendulum}

In the mid-forties it was recognized $[4,5]$ that the cyclotron would never be able to accelerate particles to energies above a critical value. The problem lies in the fact that in free space static electric or magnetic or both fields together cannot focus a beam of charged particles in all planes through the axis of the beam. Fortunately, this difficulty, which applies to linear accelerators, can be circumvented to some degree in circular systems. However, the degree of focusing permitted by the effect of the centrifugal force is extremely weak. These considerations suggested that energies higher than $10 \mathrm{MeV}$ are not attainable without unreasonable expenditures of money and materials. However, in 1949 Nick Christofilos from Athens, who at that time was an elevator operator, dreamt up the principle of strong focusing. Although he got a patent, his work was unpublished and the principle was re-discovered [6] in 1952.

The central idea of the principle of strong focusing is to use a sequence of strong focusing and defocusing fields to achieve a net focusing effect. This idea is best illustrated using the principle of the upside-down pendulum [7]. Consider the motion of a point mass on one end of a massless rod. The opposite end of the rod is connected via a hinge to another rod that is fixed to a support. This pendulum experiences a constant gravitational force pulling downwards. When the point of suspension is such that the pendulum hangs down and we concentrate on the limit of small displacements from the stable hanging position, we can approximate the motion of the mass by that of a harmonic oscillator. However, when the pendulum is upside-down and stands straight against the gravitational force, the motion is unstable and the mass tends to fall down. In this case we face an inverted harmonic oscillator of negative steepness. In order to stabilize it, Kapitza [8] in 1951 suggested a rapid vertical modulation of the foundation of the pendulum. When the modulation frequency is above a critical value determined by the length of the pendulum, the gravitational acceleration and the modulation amplitude, the motion of the pendulum becomes stable. Why is this so?

Many mathematical arguments offer themselves. They range from the method of averaging [9] via the secular growth theory [10] to the Floquet theorem [11]. However, none of these mathematical techniques provide deeper insight into the physics of the stabilizing mechanism. For the sake of argument and to illustrate this lack of insight, we follow the Floquet reasoning.

The equation of motion for the phase angle $\varphi=$ $\varphi(t)$ of the pendulum, measured from its upside-down unstable position, is the Mathieu equation [12], as discussed in the Appendix. The Floquet theorem [11] determines the domains of stability and instability of this equation. Hence, it is the stability chart [12] of the Mathieu equation that governs the parameter regime for which the upside-down pendulum is stable.

What is the mechanism of this stabilization? When the foundation accelerates the pendulum upwards, the gravitational force which pulls the pendulum down and makes its motion unstable, is reduced. A push up therefore corresponds to a stabilizing force. On the other hand, a motion of the foundation downwards increases the effective gravitational force and thereby enhances the instability of the pendulum: A pull down corresponds to a de-stabilizing force. At first sight one might think that during a complete cycle of modulation the two effects average out, however, during a majority of time there is a pushing up effect. Why is this?

In the stable mode the pendulum performs two types of motion, the secular and the micro-motion. The secular motion corresponds to a slow oscillation of the mass between two extremes of phase angles. In addition, the mass undergoes a rapid motion with a frequency identical to the modulation frequency. It is this micro-motion, superimposed on the secular motion, which makes the pendulum stable and which breaks the symmetry between the upward and the downward push. Indeed, due to the geometry of the pendulum, the angular acceleration is not only determined by the acceleration due to the modulation, 
but by the product of this acceleration and the instantaneous phase angle. The corresponding force is therefore a tidal force: No displacement of the oscillator - no force; large displacement - large force. The modulation of the foundation translates into a modulation of the phase angle. Since the effective acceleration is the product of the acceleration due to the modulation and the phase angle we find the product of two oscillatory functions with the same phase. The time average of such a quantity is a positive constant providing a harmonic oscillator potential of positive steepness for the slow motion.

\section{Paul Trap, Helium Atom and Negative Ions}

Many other applications of the upside-down pendulum come to mind, for example the Paul trap [13], that allows us to store and manipulate single ions in a controlled way. It is worth mentioning that Paul traps play an important role in the recent proposals for a quantum computer.

In the Paul trap the need for a time dependent force is dictated by the Poisson equation of electrostatics making it impossible to create binding forces in all three dimensions of space. Indeed, the Poisson equation enforces the feature that even when two spatial directions enjoy a binding potential the third one is antibinding. To overcome the instability of the trap in the third direction two possibilities offer themselves: apply a time independent homogeneous magnetic field that confines the ion in the third direction or apply an alternating voltage to the trap. The first approach corresponds to the Penning trap, the second to the Paul trap. In the most elementary case the Paul trap consists of a quadrupole field, giving rise to harmonic oscillator potentials. The alternating voltage applied to the electrodes creates a dynamical binding in three dimensions. In each dimension the physics of this binding phenomenon is identical to the upside-down pendulum.

The helium atom represents another interesting application of this concept of dynamical binding. For more than one hundred years physicists have tried to understand the motion of two electrons around the nucleus. A one-dimensional model in which the electrons move along a line with the nucleus at the origin brings out the essential physics. At first sight, a situation in which the electrons are on different sides of the nucleus seems to be preferable since in this way the electrons can avoid each other in a most effective way. However, an extremely interesting situation [14] occurs when both electrons are on the same side. Indeed, here one electron is close to the nucleus and oscillates rapidly between two equilibrium points. The second electron is far away from the nucleus and moves slowly feeling the attractive force of the nucleus and the repulsion from the inner electron. Indeed, the fast motion of the inner electron creates a time averaged repulsive potential, which superposes with the attractive Coulomb potential of the nucleus, giving rise to a local potential minimum for the outer electron. In this case, the electron-electron repulsion, together with the rapid motion of the inner electron, forms a time dependent barrier to stop the unstable motion of the outer electron caused by the attraction towards the nucleus. This phenomenon is the upside-down pendulum in disguise [15].

Our last illustration of effective potentials, arising from time averaging, is the effect of an induced dipole or multipole. It helps us to make the transition to unusual bound states in quantum mechanics. Two examples offer themselves, (i) the binding of an electron in a negative ion [16] and (ii) the atom in a standing electromagnetic wave [17]. The field of an electron can polarize a neutral atom even when the electron is at a distance large compared with the atomic dimensions. This interaction between the electron and the atom leads to a force of attraction. This attraction is the reason why some atoms, such as hydrogen or halogens, are able to form negative ions by an attachment of an electron.

We now consider the motion of an atom in a standing electromagnetic wave. Indeed, in quantum optics many such experiments have been and are being performed. The light induces a dipole moment in the atom and this dipole again interacts with the light field. Since the interaction energy is the product of the induced dipole and the field, it enters quadratically. Consequently, the position dependence of the interaction energy follows from the position dependence of the square of the field, that is from the square of the mode function thereby creating the effective potential for the motion of the atom.

\section{Energy Ground State in the Accordion Potential}

All examples in the previous sections illustrate how dynamical binding can occur in classical physics. We now turn our attention from Newton's equation of 


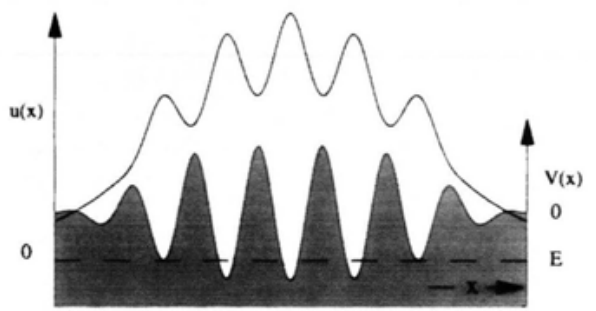

0

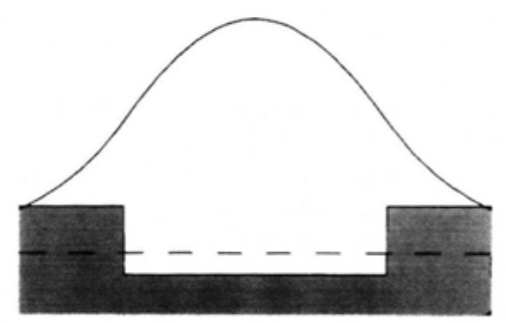

Fig. 1. Formation of a bound state in the accordion potential. This one-dimensional potential oscillates in a confined region of space and decays rapidly to a constant outside, therefore being reminiscent of an accordion. The modulations in the wave function and in the potential (top) give rise to an effective potential well (bottom) determining the envelope of the bound state.

motion

$$
\frac{\mathrm{d}^{2} \varphi(t)}{\mathrm{d} t^{2}}+\left[\xi_{0}+\xi(t)\right] \varphi(t)=0
$$

for a harmonic oscillator with time dependent steepness $\xi(t)$ to the time independent Schrödinger equation

$$
\frac{\mathrm{d}^{2} u(x)}{\mathrm{d} x^{2}}+\frac{2 M}{\hbar^{2}}[E-V(x)] u(x)=0
$$

for a point particle of mass $M$ in a position dependent potential $V(x)$; these equations are identical in form. The role of the angle coordinate of the classical upside-down pendulum is now played by the energy wave function $u$. Moreover, the time variable $t$ is replaced by the coordinate variable $x$. Indeed, Newton's equation of motion is an equation of second order in time for the position and the Schrödinger equation is an equation of second order in position for the wave function. We emphasize that this analogy between the two equations only holds true for the classical harmonic oscillator. Whereas the Schrödinger equation is always linear, a feature independent of the form of the potential, the form of Newton's equation strongly depends on the shape of the potential and in general is nonlinear.

We focus on a periodic potential in space. The particular shape of this potential is of no importance as long as it is averages out to zero over space; it is as often positive as it is negative. For our analysis it is crucial that the oscillations of the potential start at a given point in space and end thereby connecting a flat space through a domain of wiggles back to a flat space. We measure energy relative to flat space. Figure 1 shows an example of such a potential.

It is not surprising that under these circumstances we find bound states of negative energy, however, under appropriate conditions this potential can also display a localized state of zero energy. Thus, it is again the physics of the upside-down pendulum that allows us to understand this phenomenon.

We first concentrate on the one-dimensional case. Guided by the analogy between the Newton and the Schrödinger equation we expect the corresponding ground state energy wave function to exhibit a slowly varying envelope that is modulated with the period of the periodic potential; but where does the binding come from? The origin of this bound state lies in the modulation of the envelope - without the modulation there is no binding. The modulation can be easily understood: the particle is more likely to be in a stable minimum of the potential rather than in an unstable maximum.

The curvature of the wave function at a given position, expressed by its second derivative, is determined, not only by the potential, but also by the wave function at that point. Indeed, according to the Schrödinger equation (2), it is the product of the potential and the wave function that governs the curvature. This quantity contains, apart from other terms, the product of the oscillatory potential and the modulations of the wave function. The simplest case of a cosine modulation gives rise to the square of the cosine, thus creating a constant potential and a part that varies in space with twice the modulation frequency.

We focus on the constant part and note that this constant is always negative. In order to explain this feature we recall that the modulation of the envelope of the wave function is such that at the minima of the potential we have local maxima of the wave function, and vice versa. Hence, the modulation and the potential differ in their sign and their product creating the potential well is negative. The fact that the potential only wiggles over a finite domain lets this 
$\mathrm{E}<0$
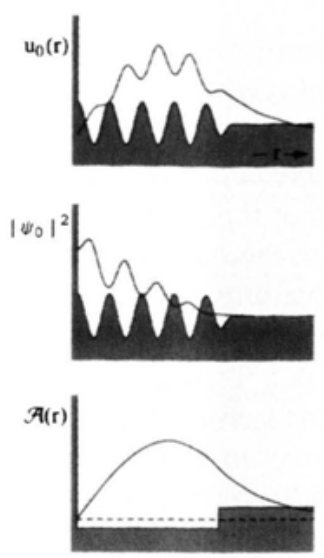

$\mathrm{E}=0$
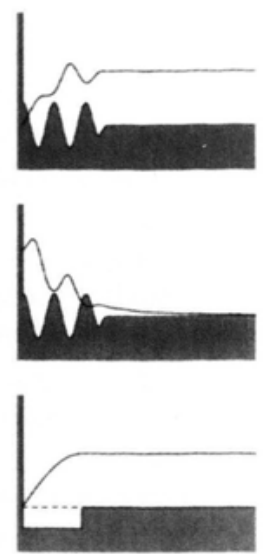

$\mathrm{E}>0$
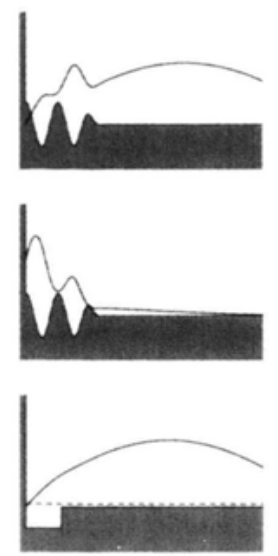

Fig. 2. Formation of a bound or localized state in a three-dimensional accordion potential. We consider the cases of the eigenenergy being smaller (left column), equal (middle column) or larger (right column) than the free space potential energy. The modulation of the potential enforces a modulation of the radial wave function (top row). In complete analogy to the one-dimensional case, the envelope of the wave function follows from the effective potential well (bottom row) created by the product of the modulations in the wave function and the potential. For negative energies the radial wave function decays exponentially in the classically forbidden regime, whereas for vanishing energy it is constant. Positive energies lead to oscillatory wave functions. In the last two cases the radial wave function is not localized. However, the probability density $\left|\psi_{0}(r)\right|^{2}=\left|u_{0}(r) / r\right|^{2}$ shown in the middle row is localized due to the additional factor $1 / r^{2}$.

constant negative potential be an attractive potential well whose width is determined by the range of the oscillations. The depth of the well involves, apart from other parameters, the square of the height of the periodic potential. For more mathematical treatment and explicit expressions for the well we refer to the Appendix.

There is a close analogy between the ground state in the periodic potential and the problem of the upsidedown pendulum. In both cases the binding effect is a consequence of the average of the product of two oscillatory functions: for the pendulum it is the time dependent modulation of the foundation and the time dependent angle of oscillation, which creates a harmonic oscillator of positive steepness. When considering the particle in the periodic potential, it is the product of the position dependent potential and the wave function modulated in space that gives rise to a well. However, there is a dramatic difference: whereas the modulation of the pendulum is on for all times, the spatial modulation is confined to a certain domain of space. It is this confinement in space, together with the creation of an effective potential, which defines the bound state.

\section{Zero-energy Ground State}

The spatial domain over which the wiggles are present and the depth of the potential well determine the ground state energy. Is it possible to choose these parameters so as to achieve a ground state of zero energy? The answer is: it depends! In one dimension the answer is a flat no, but in three dimensions it is possible, as we shall now show.

We start by considering the situation in one dimension. As we decrease the depth of the well and the energy of the ground state approaches zero, the tails of the wave function reach more and more into the forbidden region outside of the well. This feature is a consequence of the fact that the decay is governed by the square root of the absolute value of the energy. In the limit of zero energy the wave function is no longer localized; no mechanism prevents the wave from flooding all space. Consequently, there is no ground state of zero energy in one dimension.

Is it possible to construct such a zero-energy bound state in higher dimensions? In order to answer this question, we first concentrate on the threedimensional case and consider a potential $V=V(r)$ that wiggles along the radial direction. Beyond a final radius $r_{0}$, the potential vanishes. The time independent Schrödinger equation

$$
\frac{\mathrm{d}^{2} u_{0}(r)}{\mathrm{d} r^{2}}+\frac{2 M}{\hbar^{2}}[E-V(r)] u_{0}(r)=0
$$

for the radial energy wave function $u_{0}=u_{0}(r)$ corresponding to vanishing angular momentum is then identical to the one-dimensional case. Due to the product of the oscillatory potential and the wave function, and the finite domain of oscillations, we find an attractive potential well of radius $r_{0}$. There is, however, 
a subtle difference to the one-dimensional case: it is the origin where the radial wave function has to vanish [18] corresponding to an infinitely high potential wall at the origin. This feature is a consequence of the spherical symmetry. Therefore, the infinite wall at the origin provides one side of the effective well creating even a node of the wave function. The other wall of finite height is due to the effective potential together with the finite range of the oscillatory domain.

For appropriate parameters such as the final radius and the depth of the modulation of the potential we can fit a ground state of negative energy into this well, as shown in Figure 2. The corresponding wave function displays a node at the origin and an exponential decay in the classically forbidden domain beyond the critical radius, with a single maximum lying in between. Can we now take the limit of zero energy and thus realize a zero-energy ground state?

The answer is yes! Indeed, we can arrange the depth of the well such that exactly one quarter of a period of a sine oscillation fits into the potential well. Why a quarter of a period? Since the decay of the wave function outside of the potential is governed by the square root of the energy, we find that for zero energy the wave function is constant. Due to the condition of continuity of the wave function it must take on the value at the wall. The radial wave function displays a node at the origin and increases like a sine function to a constant value. However, the sine function must merge smoothly with the constant, which is only possible at a point where the sine function has an extremum. Since we want to have the ground state without a node, except the one at the origin, the only possibility for such a merger appears at one quarter of a period [19] of the sine function.

Obviously, this zero-energy ground state radial wave function does not display any localization. The localization becomes apparent when we recall that we have to divide the radial wave function by the radial variable in order to find the total wave function, that is

$$
\psi_{0}(r)=\frac{u_{0}(r)}{r} .
$$

Indeed, now the total wave function enjoys a maximum at the origin and decays with the inverse power of the radius. We emphasize however, that the total wave function is not square integrable since the volume element $4 \pi r^{2} \mathrm{~d} r$ brings in the square of the radius. This contribution cancels exactly the factor creating the localization in the probability density, that is, in the absolute value squared of the wave function. For this reason we refer to this state as localized state rather than bound state.

We conclude this discussion by briefly addressing the case of positive energies. Here, the exponential decay into the classically forbidden region of a wave function corresponding to negative energy turns into right and left running plane waves of positive energy. Obviously, plane waves are not localized wave functions. Nevertheless, the total wave function is concentrated at the origin due to the inverse power of the radius; in this sense we have localized wave functions in the continuum.

\section{Quantum Anti-centrifugal Force}

The physics of a bound state in two dimensions is very different from the one in other dimensions; and this for many reasons. In [3] we have studied this case in more detail. In the present paper we only highlight and motivate the results of [3]. For detailed derivations we refer to that article. To bring out the peculiarities of the two-dimensional case we first consider the case of no external potential.

In two dimensions a vanishing angular momentum does not imply [20] a vanishing centrifugal potential as in three dimensions. Indeed, here the radial Schrödinger equation reads

$$
\frac{\mathrm{d}^{2} u_{0}(r)}{\mathrm{d} r^{2}}+\frac{2 M}{\hbar^{2}}\left[E-V_{Q}(r)\right] u_{0}(r)=0 .
$$

We can trace the origin of the new potential

$$
V_{Q}(r) \equiv-\frac{\hbar^{2}}{2 M} \frac{1}{4 r^{2}}
$$

back to the non-vanishing commutation relation between the operators of momentum and the radial unit vector. This potential is, therefore, a true quantum potential. Since, in addition, it is attractive rather than repulsive we have named it the quantum anti-centrifugal potential [3]. Moreover, the potential is attractive only in a two-dimensional world. For one or three dimensions it vanishes, but for higher than three dimensions it becomes repulsive.

The quantum anti-centrifugal potential has brought us into a rather unusual situation. Usually, we perform wave mechanics in a classical external potential. 
In the present context however, we have a quantum potential. This feature stems from the reduction of space from three to two dimensions, reminiscent of the Born-Oppenheimer approximation in molecular physics. A molecule is a quantum system consisting of several degrees of freedom. In the framework of the Born-Oppenheimer approximation we solve the Schrödinger equation for the electrons for a fixed position of the nuclei and then use the resulting potential curves, that is, the electronic energies as a function of the separation of the nuclei to determine their relative motion. In this sense we indeed perform wave mechanics on potentials that are not due to external forces but due to quantum mechanics. In the example of the nuclear motion in a molecule, the appearance of wave mechanics in a quantum potential is a consequence of the reduction of the degrees of freedom [21].

The attractive quantum anti-centrifugal potential manifests itself even in the energy wave function of a free particle, that is in the absence of any other external potential. The ordinary Bessel functions $J_{0}$ and $N_{0}$ are two independent solutions of the time independent Schrödinger equation for positive energy. Both show a bunching of nodes towards the origin, as suggested by an attractive potential leading to an acceleration of the particle towards the origin and demonstrated for the example of $J_{0}$ in Figure 3.

We have to compare and contrast this situation with the case of a particle with one unit of angular momentum, where the centrifugal potential is indeed repulsive. It is worth noting that the quantum anticentrifugal potential reduces the strength of repulsion of the centrifugal potential, and the effective potential is not as repulsive as in the classical case. In the case of one unit of angular momentum, the solutions of the time independent Schrödinger equation are the ordinary Bessel functions $J_{1}$ and $N_{1}$. As expected by the repulsive potential enforcing a deceleration of the particle as it approaches the origin, the nodes of the Bessel functions show anti-bunching towards the origin.

One might wonder if the quantum anti-centrifugal potential is strong enough to support a bound state with negative energy. In the transition from positive to negative energies real wave numbers transform into purely imaginary ones as a consequence of the quadratic dispersion relation between energy and wave number. Therefore, the ordinary Bessel functions $J_{0}$ and $N_{0}$ turn into modified Bessel functions $I_{0}$ and $K_{0}$. Since $I_{0}$ explodes for large arguments

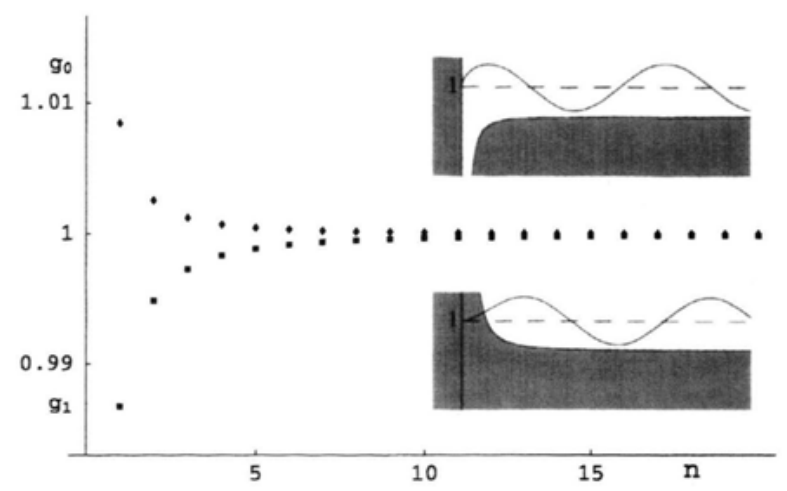

Fig. 3. Node bunching and anti-bunching of energy eigenfunctions of a free particle in a two-dimensional space. The centrifugal potential corresponding to a non-vanishing angular momentum is repulsive (bottom inset). This feature gives rise to an anti-bunching of the nodes of the energy eigenfunction $u_{1}(\rho) \equiv \sqrt{\rho} J_{1}(\rho)$, that is an antibunching of the zeros $j_{1, n}$ of the Bessel function $J_{1}$. In contrast, the centrifugal potential corresponding to a vanishing angular momentum is attractive (top inset). This feature leads to a bunching of the nodes of the wave function $u_{0}(\rho) \equiv \sqrt{\rho} J_{0}(\rho)$, that is a bunching of the zeros $j_{0, n}$ of the Bessel function $J_{0}$. As a measure $g_{m}(n) \equiv \pi / \Delta_{m}(n)$ of bunching or anti-bunching we use the inverse of the separation $\Delta_{m}(n) \equiv j_{m, n+1}-j_{m, n}$ of neighbouring zeros $j_{m, n}$ of the $m$-th Bessel function $J_{m}$ in units of the free space separation $\pi$. Squares correspond to $g_{1}(n)$ of a weakly repulsive centrifugal potential. Diamonds represent $g_{0}(n)$ with an attractive centrifugal potential - the quantum anti-centrifugal potential. The physics of the non-relativistic free particle does not contain an intrinsic unit of length. When we define a dimensionless length $\rho \equiv k r$ where $k$ is the wave number, the dimensionless energy eigenvalue is unity.

[12], that is, large radial distances, this solution does not satisfy the requirement of exponential decay enforced by the attractive quantum anti-centrifugal potential. Only the function $K_{0}$ achieves this goal, but it has another disease: it explodes at the origin like a logarithm.

This singularity at the origin indicates that the solution

$$
u_{0}(r)=\sqrt{\frac{k}{\pi}} \sqrt{k r} K_{0}(k r)
$$

of the Schrödinger equation, shown in Fig. 4, does not describe a truly free particle but that there is a delta function potential present. The strength of this external potential determines [22] the energy eigenvalue of this bound state. Moreover, due to the quantum anti-centrifugal potential the probability of finding the 


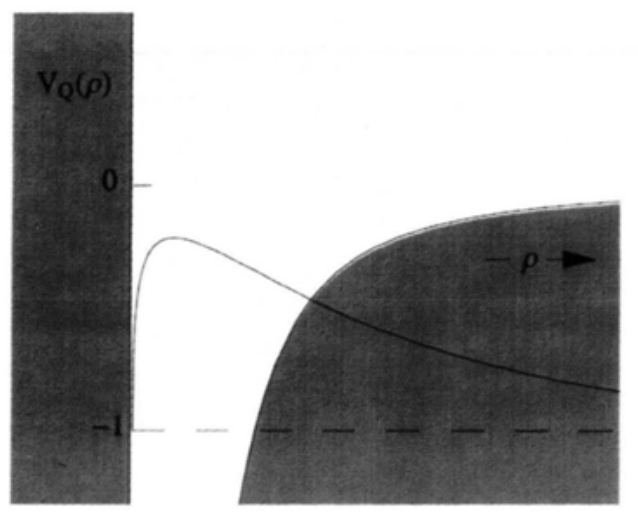

Fig. 4. Bound state of a "free" particle. The attractive quantum anti-centrifugal potential $V_{Q}$ supports a single bound state of negative dimensionless energy. The corresponding energy wave function $u_{0}(\rho) \equiv \sqrt{\rho / \pi} K_{0}(\rho)$ is normalizable since it decays exponentially into the classically forbidden regime provided by the quantum anti-centrifugal potential. Here $K_{0}$ is the Neumann function of purely imaginary argument. At the origin the wave function has infinite steepness indicating the presence of an additional potential located solely at the origin, that is a delta function potential.

particle within a given area in space is concentrated along a band around the location of the delta function; a remarkable feature, unique to two dimensions. In a similar arrangement in one or three dimensions the maximum of the probability is at the location of the potential minimum. This property might have interesting applications for guiding atoms along wires [23] or electro-magnetic waves in wave-guides [24].

We now return briefly to the case of an energy eigenstate in a two-dimensional oscillatory potential of finite range. As emphasized in the previous paragraph, the case of vanishing angular momentum contains the most quantum effects. Here, the ground state wave function of negative energy feels the combination of the quantum anti-centrifugal potential and the potential well created by the product of the wave function and the potential.

\section{Light Induced Potentials, Trojan Wave Packets and the Lone Electron}

The examples discussed in the preceding sections represent but only a small subsection of the class of unusual bound states. They have been selected because they follow or are motivated by the physics of the upside-down pendulum. However, there are many more intriguing bound states in atomic and molecular physics [25]. We conclude our paper by briefly summarizing three examples starting with one that is still closely related to our main theme of the upside-down pendulum and gradually moving away towards new frontiers.

Consider a diatomic molecule with various electronic states. Within the Born-Oppenheimer approximation the electronic states provide the potentials for the relative motion of the two nuclei. A strong external time dependent laser field interacts with the electrons and couples the individual electronic potential surfaces. The wave functions for the vibratory motion of the nuclei are therefore coupled through a time dependent periodic interaction Hamiltonian. The product of the interaction Hamiltonian and the nuclear wave functions governs the time evolution of the wave functions; a feature that is again reminiscent of the upside-down pendulum. In the latter the product consists of the time dependent steepness of the oscillator and the phase angle. The only difference lies in the fact that in the classical case the Newton equation is of second order, whereas the Schrödinger equation is of first order. However, this distinction is superficial since we are dealing with the nuclear wave functions corresponding to different electronic states and hence, with a system of differential equations of first order.

Due to this product of two periodic functions with the same period (interaction Hamiltonian and wave function) we find again a constant which lifts up the electronic potential by integer multiples of the energy of the absorbed photon. In mathematical terms this energy elevation is a consequence of Floquet states. However, it can be viewed as just one more illustration of the upside-down pendulum with counter-intuitive consequences. Indeed, when we consider a binding and a repulsive potential, this constant shift in energy can result in a crossing of the potential curves. Since in a diatomic molecule there exists a non-crossing rule, we obtain an avoided crossing forming new potentials; one is binding and one is repulsive. However, these potentials are rather peculiar because each of them consists of parts of the two original potentials. The formation of new bound states [26] in this light induced molecule has been observed experimentally [27].

Trojan asteroids are celestial bodies that are held in their positions by the gravitational forces of the sun and Jupiter. A similar phenomenon can occur [28] in an atom where the electron plays the role 
of a Trojan asteroid, and the nucleus substitutes for one of the two planets. A circularly polarized electromagnetic field simulates the effect of the second planet. Indeed, the electromagnetic interaction of the electron with the field and the Coulomb attraction between the electron and the nucleus replace the gravitational attraction of the celestial bodies. In a frame rotating with the electromagnetic field, the motion of the electron is in a plane and governed by three potentials which depend on the two-dimensional radial direction: the Coulomb attraction of the nucleus, the centrifugal potential of the circular motion and the linear potential arising from the interaction of the electron with the electromagnetic field. For short distances the Coulomb potential dominates, whereas for large distances the repulsive potential of the circular motion prevails. Consequently, for intermediate distances an unstable potential maximum occurs. This feature is in complete analogy with the upside-down pendulum. But where does the stabilizing drive come from? The answer to this question lies in the fact that the radial motion is coupled to the angular motion. The latter is locked to the rotating electromagnetic field, and the electron performs angular vibrations around this stable point of equilibrium. These vibrations couple into the radial motion creating a dynamically binding potential. This classical picture also holds true for the quantum case as verified by extensive studies of wave packets propagating in this minimum.

In our last example of unusual bound states it is the balance of static forces that is responsible for the formation of a bound state of a lone electron in a Rydberg atom in crossed electric and magnetic fields [29]. The crossed-field situation is of particular appeal in the field of quantum chaology since in this system energy is the only conserved quantity. The classical system displays chaos and is therefore of interest in the search for fingerprints of chaos in the corresponding quantum system. Apart from these questions of quantum chaos there is another quite interesting aspect of this system. The electron in the atom experiences not only the attractive Coulomb potential of the nucleus, but also a linear potential due to a constant electric field and a binding harmonic oscillator potential due to the magnetic field. The superposition of all three potentials creates a local potential minimum far away from the nucleus. An electron bound in this minimum displays a large dipole moment that can be observed by sending the atom through an inhomogeneous electric field. Indeed, the experiments [23] have confirmed the existence of this far outside lying minimum.

We have come a long way on our journey into unusual bound states. Starting from the classical physics of the upside-down pendulum we have been led to the phenomenon of a zero-energy ground state in a periodic potential in three dimensions. The two-dimensional world has even more surprises in store: the attractive quantum anti-centrifugal force that manifests itself in a free particle through the bunching of the nodes or even in a bound state. Here the probability of finding the particle is concentrated in the domain where no force is acting. This feature resulting from the action of the quantum anti-centrifugal potential could be useful in guiding atoms along wires or electromagnetic waves along fibers. Experiments on trapping cold atoms by single photons [30] or along a whispering gallery mode of a glass sphere resonator [31] are yet more realizations of the physics of the upside-down pendulum and give us confidence that our predictions of unusual bound and localized states can be verified experimentally in the near future.

\section{Acknowledgements}

This work started when I (WPS) had the great privilege to be a postdoc with John A. Wheeler more than a decade ago. Since that time, John and I have frequently returned to the topics addressed in the present paper. These discussions took place in trains, planes and automobiles and at various locations such as Hightstown, High Island, and Ulm. I thank John for this wonderful time, his outstanding hospitality at Hightstown and High Island and especially for the unique experience to work with him. The present paper is partially based on notes and sketches of figures prepared jointly. I am grateful for many fruitful discussions and John's deep insights and, in particular, for allowing us to use the material that had originally been obtained in close collaboration with him. The proceedings of the Lake Garda Conference are a most welcome opportunity to finally summarize this project started many years ago. Moreover, we are grateful to I. Białynicki-Birula for a critical reading of the manuscript. Two of us (MAC and WPS) thank R. Bonifacio for his hospitality and for organizing a most interesting conference in the splendid surroundings of Lake Garda. The work of WPS is partially supported by the Deutsche Forschungsgemeinschaft. Moreover, he gratefully acknowledges a travel grant 
from the Universität of Ulm which made part of this research possible.

\section{Appendix}

In contrast to the main body of the paper, we pursue in the present Appendix a more mathematical approach. In particular, we emphasize the similarities between the classical equation of motion for the upside-down pendulum and the Schrödinger equation for a nonrelativistic particle in a periodic potential. For this purpose, we first cast the corresponding equations into a dimensionless form. This approach allows us to simultaneously derive equations for the dynamics contained in the macro- and micro-motion of the pendulum or the envelope function and the modulation of the ground state wave function.

We start by summarizing the classical equation of motion for the upside-down pendulum. For the sake of simplicity we consider the limit of small angles $\varphi$ measured relative to the vertical position.

A vertical acceleration $\ddot{f}$ of the foundation translates into a vertical acceleration of the mass $M$ of the pendulum and adds to the gravitational acceleration $g$ pointing downwards. Here, the sign of $\ddot{f}$ is crucial: when $\ddot{f}$ is positive, that is, when the pendulum is accelerated upwards, the gravitational acceleration pointing downwards is reduced. Likewise, when $\ddot{f}$ is negative, that is, when the pendulum is accelerated downwards, the gravitational acceleration is increased. Consequently, the total acceleration is $g-\ddot{f}$. Hence for small angles $\varphi$ the force tangentially to the rod of the pendulum of length $L$ reads

$$
M L \frac{\mathrm{d}^{2} \varphi}{\mathrm{d} t^{2}}=M(g-\ddot{f}) \varphi,
$$

giving rise to

$$
\frac{\mathrm{d}^{2} \varphi(t)}{\mathrm{d} t^{2}}+\left[-\Omega^{2}+\frac{1}{L} \ddot{f}\right] \varphi(t)=0,
$$

where $\Omega^{2} \equiv g / L$.

It is reasonable to assume that the modulation $f$ is a periodic function of period $T \equiv 2 \pi / \nu$. This time scale allows us to introduce a dimensionless variable $\theta \equiv \nu t$, and the differential equation of the upsidedown pendulum takes the form

$$
\frac{\mathrm{d}^{2} \varphi}{\mathrm{d} \theta^{2}}+[\kappa+a(\theta)] \varphi=0 .
$$

Here we have introduced the abbreviations $\kappa \equiv$ $-(\Omega / \nu)^{2}$ and $a(\theta) \equiv f^{\prime \prime}(\theta) / L$ for the steepness of the inverted harmonic oscillator and the acceleration due to the modulation, respectively. Moreover, prime denotes differentiation with respect to $\theta$.

We now make the connection to the time independent Schrödinger equation

$$
\frac{\mathrm{d}^{2} u(x)}{\mathrm{d} x^{2}}+\frac{2 M}{\hbar^{2}}[E-V(x)] u(x)=0
$$

for a particle of mass $M$ in the potential $V(x)$, which is periodic in space with period $\lambda \equiv 2 \pi / k$.

When we introduce the recoil energy $E_{r} \equiv$ $(\hbar k)^{2} /(2 M)$ and the dimensionless coordinate $\theta \equiv$ $k x$, the Schrödinger equation takes the form

$$
\frac{\mathrm{d}^{2} u}{\mathrm{~d} \theta^{2}}+[\eta-v(\theta)] u=0,
$$

where the dimensionless energy eigenvalue $\eta \equiv$ $E / E_{r}$ and the potential $v(\theta) \equiv V(\theta / k) / E_{r}$ are scaled in units of the recoil energy.

The two dimensionless equations of the driven pendulum (10) and the quantum particle in the periodic potential (12) have identical structure. In Sects. 2 and 4 we have presented qualitative arguments to explain the motion of the pendulum as a superposition of a slow motion (macromotion) and a rapid motion (micromotion) or, in the language of wave functions, to decompose the energy wave function into an envelope and a modulation. We now take advantage of the analogy between the two systems to support these qualitative arguments by rigorous mathematics. For this purpose we start from the equation

$$
u^{\prime \prime}(\theta)+[\eta-v(\theta)] u(\theta)=0 .
$$

Again, we emphasize that $u$ can either be the phase angle of the pendulum or the wave function. Likewise, $\theta$ represents time or position and the drive $v(\theta)$ is either the acceleration of the foundation or the potential.

In order to find the solution of (13), we make the Ansatz

$$
u(\theta)=\mathcal{A}(\theta)[1-\epsilon v(\theta)],
$$

consisting of the product of the slowly varying envelope $\mathcal{A}(\theta)$ and the modulation caused by the drive $v(\theta)$. 
We substitute the Ansatz (14) into (13), which yields

$$
\begin{aligned}
\mathcal{A}^{\prime \prime}+\left[\eta+\epsilon v^{2}\right] \mathcal{A} & -\left[\mathcal{A}^{\prime \prime}+\left(\eta+\frac{1}{\epsilon}+\frac{v^{\prime \prime}}{v}\right) \mathcal{A}\right] \epsilon v \\
& -2 \epsilon \mathcal{A}^{\prime} v^{\prime}=0 .
\end{aligned}
$$

So far, our analysis is exact. We now solve the equation (15) in an approximate way. For this purpose, we first neglect the last contribution in (15) involving the product of the first derivatives of the envelope function and the potential. This approximation is justified since $v^{\prime}$ is out of phase with $v$ and therefore cannot lead to a significant contribution. Moreover, we choose $\epsilon$ such that

$$
\frac{1}{\epsilon}+\frac{v^{\prime \prime}}{v}=0
$$

We emphasize that this is not possible in a strict sense, since $v^{\prime \prime} / v$ is not necessarily a constant. However, in the oscillatory domain of the potential the most elementary model $v(\theta) \propto \cos (\theta)$ suggests the estimate $v^{\prime \prime} / v \simeq-1$, and hence we find

$$
\epsilon=+1 \text {. }
$$

Provided the envelope $\mathcal{A}$ satisfies the equation

$$
\mathcal{A}^{\prime \prime}+\left[\eta+\epsilon v^{2}\right] \mathcal{A}=0,
$$

also the second contribution in (15) vanishes to this order in $\epsilon$. This is due to the fact that the second contribution is already multiplied by $\epsilon$.

For the case of the upside-down pendulum, the parameter $\eta$ is negative. In the absence of the drive, the negative value of $\eta$ leads to an exponential growth of

[1] P. Ehrenfest, Naturwiss. 11, 543 (1923); for an excellent introduction into and comprehensive summary of the early quantum mechanics we refer to D. ter Haar, The Old Quantum Theory, Pergamon Press, Oxford 1967 , p. 44. A classic in this field is M. Born, Atommechanik, Springer, Heidelberg 1925; see also J. Duck and E. C. G. Sudarshan, 100 Years of Planck's Quantum, World Scientific, Singapore 2000.

[2] J. A. Wheeler, in C. and B. S. De Witt (eds), Relativity, Groups and Topology, Gordon and Breach, New York 1964.

[3] M. A. Cirone, K. Rzazewski, W. P. Schleich, F. Straub, and J. A. Wheeler, to be published.
$\mathcal{A}$. However, the square of the drive is always positive and can create an overall positive coefficient in front of $\mathcal{A}$, providing an oscillation rather than an exponential explosion.

In the case of the quantum particle, it is advantageous to write the equation for $\mathcal{A}$ in the form of a Schrödinger equation

$$
\mathcal{A}^{\prime \prime}(\theta)+\left[\eta-v_{\text {eff }}(\theta)\right] \mathcal{A}(\theta)=0 \text {. }
$$

Here we have averaged the square of the potential over a period of the oscillations giving rise to a smooth effective potential

$$
v_{\text {eff }}(\theta) \equiv-\frac{1}{2 \pi} \int_{\theta}^{\theta+2 \pi} \mathrm{d} \theta^{\prime} v^{2}\left(\theta^{\prime}\right) .
$$

We note that this potential is always negative. When the modulation of the potential is confined in space, for example by a window function $\mu(\theta)$, and the potential $v(\theta)$ is of the form

$$
v(\theta) \approx \mu(\theta) C(\theta),
$$

where $C$ is a periodic function, we can approximate the effective potential by a potential well

$$
v_{\text {eff }}(\theta)=-\frac{1}{2} \mu^{2}(\theta)
$$

In this case, the envelope $\mathcal{A}$ is an energy wave function of this potential well, and the parameter $\eta$ is the dimensionless energy eigenvalue. Hence, $\eta$ is not determined from the outside, but rather by the well (22) itself. If the well is deep enough, it can support, apart from the ground state, also excited states with negative energy.

[4] For a summary of the problem of acceleration of charged particles to high energies see the article by J. P. Blewett, in E. U. Condon and H. Odishaw (eds.), Handbook of Physics, McGraw-Hill, New York 1958, Chapt. 9, p. 153.

[5] J. A. Wheeler in many discussions with one of us (WPS) repeatedly credited $\mathrm{H}$. Bethe with the discovery of the problems leading to the principle of strong focusing. Unfortunately, we have not been able to locate the appropriate reference. We have talked to Prof. Bethe, but also he was not able to point out the article.

[6] E. D. Courant, M. S. Livingston, and H. S. Snyder, Phys. Rev. 88, 1190 (1952). 
[7] For a treatment of the upside-down pendulum and the double pendulum in terms of scalar and matrix continued fractions see Th. Leiber and H. Risken, Phys. Lett. A 129, 214 (1988).

[8] L. D. Landau and E. M . Lifshitz, Mechanics, Pergamon Press, Oxford 1969, p. 93-95.

[9] N. N. Bogoljubov and Yu. A. Mitropolsky, Asymptotic Methods in the Theory of Non-linear Oscillations, Hindustan Publishing, Delhi 1961.

[10] Y. Kevorkian, and J. D. Cole, Perturbation in Applied Mathematics, Springer, Heidelberg 1981.

[11] E. T. Whittaker and G. N. Watson, A Course of Modern Analysis, Cambridge University Press, Cambridge 1973.

[12] M. Abramowitz and I. A. Stegun, Handbook of Mathematical Functions, Dover Publications, New York 1965; for a particularly illuminating method to motivate the stability chart of the Mathieu equation using WKB wave functions, see M. J. Richardson, Amer. J. Phys. 39, 560 (1971).

[13] W. Paul, Rev. Mod. Phys. 62, 531 (1990). A pedagogical introduction into the physics of Paul traps emphasizing the concept of the effective potential is given by P. E. Toschek, in G. Grynberg and R. Stora (eds), New trends in atomic physics, North-Holland, Amsterdam 1984, p. 390. For a summary of current activities in Paul traps see H. Walther, in B. Bederson and H. Walther (eds.), Advances in Atomic, Molecular and Optical Physics, Academic Press, Boston 1995.

[14] K. Richter and D. Wintgen, Phys. Rev. Lett. 65, 1965 (1990); J. Phys. B 24, L565 (1991): for an overview of the full classical and quantum dynamics of two-electron systems see K. Richter, G. Tanner, and D. Wintgen, Phys. Rev. A 48, 4182 (1993).

[15] We can also interpret the system of the two electrons in the helium atom as one realization of the Fermi accelerator. In the most elementary version of this device an oscillating wall confines the unbounded motion of a particle in a linear potential. For a review of the Fermi accelerator see F. Saif, I. Białynicki-Birula, M. Fortunato, and W. P. Schleich, Physics Reports, to be published.

[16] L. D. Landau and E. M. Lifshitz, Quantum Mechanics, Non-Relativistic Theory, Pergamon Press, Oxford 1965 , p. 267; There exists a large amount of literature on the physics of negative ions. See for example the classic book H. Massey, Negative Ions, Cambridge University Press, Cambridge 1976. For the most recent activities in this field see the Springer series, Production and Neutralization of Negative Ions and Beams. A similar effect appears also for an electron in the field of a super-heavy nuclei, see for example F. G. Werner and J. A. Wheeler, Phys. Rev. 109, 126 (1958).

[17] For summary see C. N. Cohen-Tannoudji, in J. Dalibard et al. (eds), Fundamental Systems in Quantum Optics, Elsevier Science, Amsterdam, 1992; see also the Nobel Lectures by S. Chu, C.N. Cohen-Tannoudji, and W. D. Phillips, Rev. Mod. Phys. 70, 685 (1998).
[18] For a most striking and counter-intuitive bound state resulting from the peculiar behavior of the radial wave function at the origin of three-dimensional space we refer to J. Rauch and M. Reed, Comm. Math. Phys. 29, 105 (1973); and M. Reed, and B. Simon, Methods of Modern Mathematical Analysis II, Academic Press, New York 1975. In this example the potential is a sequence of appropriately constructed steps that lead continuously downwards as the radial variable increases. Classically a particle of given energy has to fall down the steps. However, the reflections of the quantum wave at the individual steps interfere in a way as to localize the particle. Likewise, the same authors discuss a potential consisting of an infinite sequence of potential spikes that classically would keep a particle trapped, however, due to the tunneling effect the quantum particle escapes.

[19] A similar reasoning appears [2] in general relativity, that is in geometrodynamics, when we determine the metric coefficients of a system. They follow from Brill's equation [2], which is similar to the time independent Schrödinger equation for zero energy. In contrast to quantum mechanics where the Schrödinger equation determines the energy eigenvalues we now have to solve the equation under the constraints that the wave is not allowed to have nodes and corresponds to zero energy.

[20] S. Flügge, Practical Quantum Mechanics, Springer, Heidelberg 1971.

[21] This observation is in complete accordance with a recent paper arguing that the time dependent Schrödinger equation is an approximation of the time independent Schrödinger equation resulting from the elimination of degrees of freedom. See for example J. S. Briggs and J. M. Rost, EPJD 10, 311 (2000).

[22] K. Wódkiewicz, Phys. Rev. A. 43, 68 (1991).

[23] J. Denschlag, D. Cassettari and J. Schmiedmayer, Phys. Rev. Lett. 82, 2014 (1999).

[24] R. E. Collin, Field Theory of Guided Waves, McGraw Hill, New York 1960 p. 477.

[25] Two examples illustrate the unusual bound states that originate from the mutual interaction of the electrons in a heavy atom: The Thomas-Fermi potential together with the centrifugal potential can form a second potential minimum, which is very deep and located close to the nucleus. This effect occurs provided the atomic number is larger than 57 and we are dealing with an energy eigenstate corresponding to the angular momentum quantum number $l=3$, see for example M. Goeppert-Mayer, Phys. Rev. 60, 184 (1941). Since A. Sommerfeld we associate the motion of an electron in an atom with an ellipse or a circle. However, the electron at the top of the sea of filled atomic states moves in an effective screened potential giving rise to a necklace orbit as pointed out by J. A. Wheeler, in E. H. Lieb et al. (eds), Studies in Mathematical Physics, Princeton University Press, Princeton 1976, p. 383 .

[26] J. Bestle, Stabilisierung zweiatomiger Moleküle in intensiven Laserfeldern, Shaker Verlag, Aachen 1997. 
[27] K. Sändig, H. Figger, and T. W. Hänsch, Phys. Rev. Lett. 85, 4876 (2000).

[28] I. Białynicki-Birula, M. Kaliński and J. H. Eberly, Phys. Rev. Lett. 73, 1777 (1994).

[29] M. Fauth, H. Walther, and E. Werner, Z. Phys. D 7 , 293 (1987); G. Raithel, M. Fauth, and H. Walther, Phys. Rev. A. 47, 419 (1993).
[30] C. J. Hood, T. W. Lynn, A. C. Doherty, A. S. Parkins, and H. J. Kimble, Science 287, 1447 (2000); P. W. H. Pinkse, T. Fischer, P. Maunz, and G. Rempe, Nature (London) 404, 365 (2000).

[31] D. W. Vernooy and H. J. Kimble, Phys. Rev. A 55, 1239 (1997): ibid. 56, 4287 (1997). 